\title{
A Selective Ink Deposition Method for the Cost-Performance Optimization of Inkjet-Printed UHF RFID Tag Antennas
}

\author{
J. Virtanen, ${ }^{1}$ J. Virkki, ${ }^{1}$ A. Z. Elsherbeni, ${ }^{2}$ L. Sydänheimo, ${ }^{1}$ and L. Ukkonen ${ }^{1}$ \\ ${ }^{1}$ Department of Electronics, Rauma Research Unit, Tampere University of Technology, 33720 Tampere, Finland \\ ${ }^{2}$ Department of Electrical Engineering, University of Mississippi, Oxford, MS 38677, USA \\ Correspondence should be addressed to J. Virtanen, juha.virtanen@tut.fi
}

Received 27 January 2012; Accepted 14 March 2012

Academic Editor: Fan Yang

Copyright ( $) 2012$ J. Virtanen et al. This is an open access article distributed under the Creative Commons Attribution License, which permits unrestricted use, distribution, and reproduction in any medium, provided the original work is properly cited.

\begin{abstract}
A selective ink deposition method is proposed for fabricating inkjet-printed passive UHF RFID tag antennas with optimized costperformance ratios. The deposition method is based on identifying areas with high surface current densities on a given tag antenna and applying additional silver nanoparticle ink onto such areas to increase tag read range. In this paper, the proposed method is experimentally verified by utilizing the method on a small dipole tag antenna. Several ink deposition schemes are created, and their read range performances and ink consumptions are presented.
\end{abstract}

\section{Introduction}

Inkjet-printing technology is a prominent technique of manufacturing low-cost passive ultra-high frequency (UHF) radio frequency identification (RFID) tag antennas using inks with metal content. Inkjet printing provides a fully additive, noncontact deposition method and thus lowers production costs by minimizing material waste [1] and by allowing fast mass customization [2]. Furthermore, inkjetprinting technology enables the use of ultra-low cost and environmentally safe materials, for example, paper and wood, as substrates in diverse electronic applications.

One of the possible applications of inkjet-printing technology in electronics is printable antennas, especially RFID tag antennas as inkjet printing could lower tag manufacturing costs and allow tags to be printed directly onto product packages. Thus, inkjet-printed antennas have been developed for various kinds of RFID tags in [3-8] and for wireless sensor networks in $[9,10]$. At the moment, despite the obvious benefits and possible cost savings of inkjet-printed antennas, their cost remains too high due to the high cost of conductive inks.

A special feature of additive fabrication methods is their ability to vary the amounts of materials used to form structures on the substrate. Significant material savings are therefore possible by selectively depositing materials to areas within the design that gain the greatest benefits from it, for example, in the form of increased performance.

In the case of antenna structures, such selective deposition of materials, mainly metals, can be done in three ways. In the first approach, the antenna structure can be printed using multiple overlapping layers of conducting ink, as in [11], to create tags with varying read ranges and costs. In [11], the read range, that is, the operating range of the tags, was greatly improved with this method.

In the second approach, solid antenna structures can be divided into grids, as in [12]. In [12], material savings in excess of $50 \%$ are reported. However, dividing the antenna into grids will change the input impedance of the antenna and eventually decrease the power transfer between the antenna and RFID IC. Furthermore, the effects of this method on the performance of the antenna and in the read range of the tag are not known.

A third, more sophisticated approach is to add conductive metallization to areas with high current density. In [13], the authors have used flexographic printing to increase the thickness of the conducting ink layer in selective areas with supposedly high surface currents. This has also led to great savings in the ink consumption. This method does not cause any additional impedance mismatching, eliminating the need for retuning the antenna impedance after optimization. 
This paper presents a further developed version of the latter approach especially for inkjet-printed tag antennas fabricated using silver nanoparticle ink. The developed selective ink deposition method relies on high-precision simulations to identify areas in the antenna structure with sufficiently high surface current density on a given tag. As the method relies on results from a full-wave $3 \mathrm{D}$ simulator, the antenna geometry, on which the deposition method is utilized, can be extremely complex. Furthermore, the positional accuracy of individual droplets deposited by and inkjet-printed is excellent and allows pixels to be printed with micrometer precision. The method is demonstrated with a case example, where the ink consumption to fabricate a high performance dipole-type tag antenna is optimized.

\section{Ink Deposition Method for Cost-Performance Optimization}

The need for methods reducing the amount of conductive ink to produce well performing inkjet-printed UHF RFID tag antennas is caused by the intrinsic property of the fabrication technology. Typically, the thicknesses of conductors formed by inkjet printing are well below the skin depth of the typical printed metals at the global $860 \mathrm{MHz}$ to $960 \mathrm{MHz}$ UHF RFID band. This leads to poor radiation efficiency due to power losses in the conductors.

Power losses in an arbitrary conductor are proportional to the square of the current flow through the conductor

$$
P_{\text {loss }}=\int_{S} R I^{2} \cdot d S
$$

The resistance of the conductor is related to the resistivity of the conductive material, to the length of the conductor and to its cross-sectional area. At high frequencies, the effective conducting cross-sectional area of the conductor is decreased due to the skin effect. Due to this effect, the current travels almost completely at the surface of the conductor. The depth, at which the amplitude of the electric field has decayed by $1 / e$, is known as the skin depth of the conductor. The skin depth of a conductor can be calculated as [14]

$$
\delta=\sqrt{\frac{2}{\omega \mu \sigma}}=\sqrt{\frac{\rho}{\pi f \mu}},
$$

where $\omega$ is the angular frequency, $\mu, \sigma$, and $\rho$ are the permeability, conductivity and resistivity of the conducting material. For a conductor to have minimal losses due to the skin effect, its thickness needs to be around four to five times as thick as the skin depth of the conducting material.

For example, the skin depth of the silver nanoparticle ink used in the experiments made for this study at $900 \mathrm{MHz}$ is $3.4-4.1 \mu \mathrm{m}$ according to the data provided by the ink manufacturer [15]. Inkjet-printed conductors are usually at around 0.5 to $2 \mu \mathrm{m}$ in thickness $[16,17]$, depending on the process parameters such as the type of ink and substrate, printing resolution, and so forth. Therefore, in order to fabricate tag antennas with good radiating efficiencies, multiple overlapping layers of ink need to be used. This will lead to high productions costs if the whole antenna structure is printed multiple times.

The power losses in the antenna can be minimized without printing the whole antenna structure using multiple layers, additional metal needs to be added only to areas with high surface current levels. In order to optimize the ink consumption, these areas with high surface currents need to be identified as accurately as possible. For this purpose, a full-wave 3D electromagnetic field simulator is utilized. The simulator is used to plot the magnitude of the complex surface current on the surface of any arbitrary planar antenna suitable for inkjet printing. Choosing to plot the complex magnitude of the surface current allows for a quick way of plotting the amplitudes of the apparent surface current density vectors. Therefore, layouts for inkjet printing are obtainable directly from a single image.

The complex surface current is plotted at the design frequency of the tag antenna. It is crucial that the tag antenna's metallization is modeled using the electrical properties of the ink used in the process since it will have an effect on the current distribution on the surface of the antenna.

After the surface current distribution has been plotted, the resulting plot is used as a printing mask for the printer to add ink to these areas. The key research problem at this stage is to determine a sufficient magnitude of surface current density which is used as a printing mask to optimize the radiation efficiency and ink consumption. This research problem is addressed in Section 3, where multiple printing masks are created, each based on different surface current density levels, to discover the optimal mask pattern.

Inkjet-printing technology is perfectly suited for this type of deposition method since individual drops of ink can be placed on the substrate with great accuracy. For example, the positional repeatability of the printer used in the experiments made for this study is $\pm 25 \mu \mathrm{m}$. Therefore, the printing masks which selectively add ink can be created using high resolution. As such, the thickness of the resulting antenna can be controlled pixel by pixel.

\section{Experimental Verification}

This section presents the experimental verification of the selective deposition method in order to optimize the performance of a typical tag antenna while minimizing the amount of ink required to fabricate the antenna.

3.1. Test Antenna Structure. A short dipole-type tag antenna, shown in Figure 1, was chosen for these experiments as it represents a typical antenna found in passive UHF RFID tags. The antenna is attached to a passive Higgs-3 RFID IC from Alien Technology [18].

The test tag antenna will be printed on top of $51 \mu \mathrm{m}$ thick $200 \mathrm{HN}$ Kapton film [19]. Kapton is a low-loss, polyimide film which provides a smooth, heat-resistant surface for high precision inkjet printing. The test tag antenna is equipped with a T-match, an impedance matching structure [20], to match the input impedance of the tag antenna to the complex conjugate of the RFID IC input impedance. The T-matching 


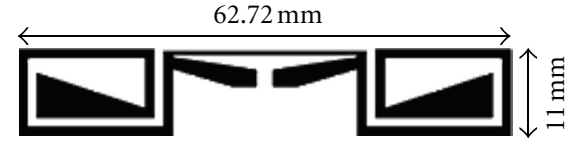

FIGURE 1: Tag antenna layout used in the experiments.
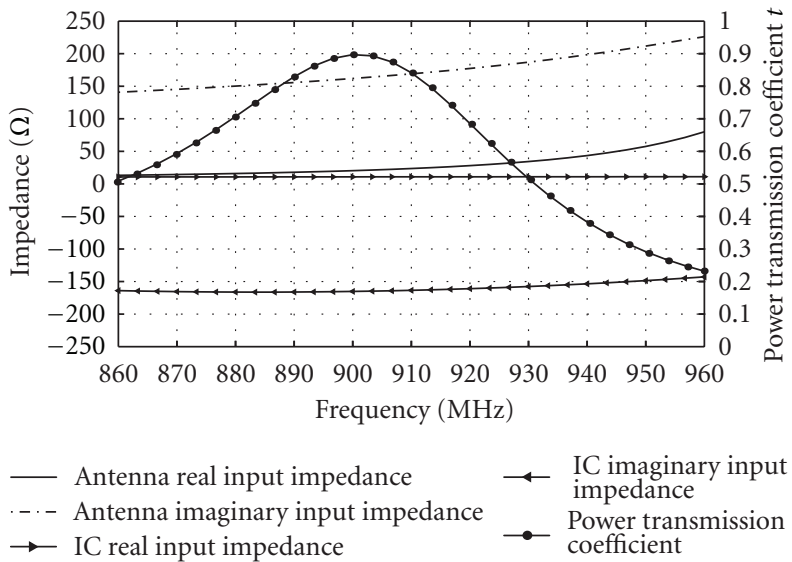

FIGURE 2: Simulated tag antenna input impedances and power transmission coefficient between the IC and tag antenna.

network is thus used to provide maximal power transfer between the IC and tag antenna [21]. The magnitude of the power transfer is described by a power transmission coefficient.

The power transmission coefficient illustrates the amount of power loss due to improper impedance matching between the tag antenna and RFID IC [22]. The power transmission coefficient $\tau$ can be calculated by

$$
\tau=\frac{4 \operatorname{Re}_{A} \operatorname{Re}_{\mathrm{IC}}}{\left|Z_{\mathrm{A}}+Z_{\mathrm{IC}}\right|^{2}}
$$

where $\operatorname{Re}_{A}$ and $\mathrm{Re}_{\mathrm{IC}}$ are the real parts of the tag antenna and RFID IC, respectively. $Z_{\mathrm{A}}$ and $Z_{\mathrm{IC}}$ are the corresponding input impedances of the antenna and IC. The simulated input impedances of the antenna and Higgs-3 IC, obtained from [23], as well as the resulting power transmission coefficient are shown in Figure 2.

The simulations were performed using Ansoft HFSS 13 [24], a finite element method-based, 3-D full-wave electromagnetic simulator software. The ink layer thickness in these simulations was set to $1.5 \mu \mathrm{m}$ (uniform) and the conductivity of the ink to $20 \mathrm{MS} / \mathrm{m}$. Kapton $200 \mathrm{HN}$ was simulated using a relative permittivity of 3.5 and a loss tangent of 0.002 .

The test antenna is optimally matched around $880 \mathrm{MHz}$ to $920 \mathrm{MHz}$ to the input impedance of IC. Figure 3 shows the simulated directivity, gain, realized gain, and radiation efficiency of the tag antenna.

The simulated radiation efficiency is only approximately 0.25 at the optimal operating frequency band. This indicates that the thickness of the conductor is not sufficient to provide low-loss operation if only a single layer of ink is deposited.

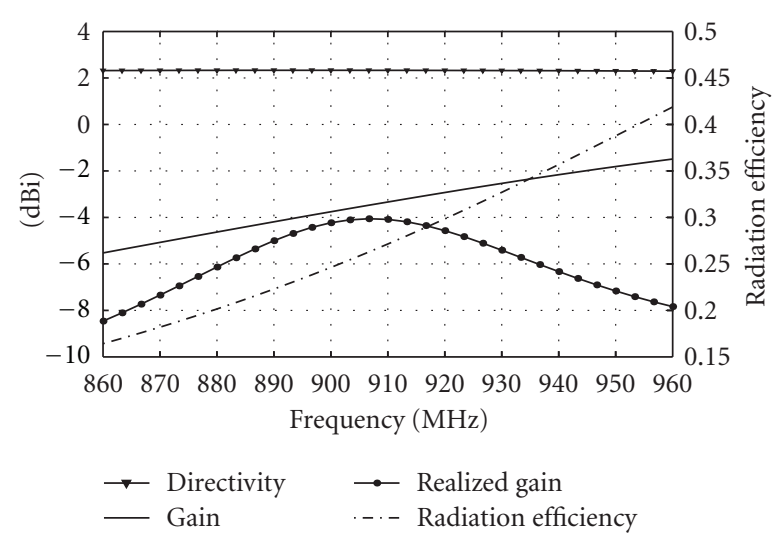

FIGURE 3: Simulated tag antenna directivity, gain, and radiation efficiency.

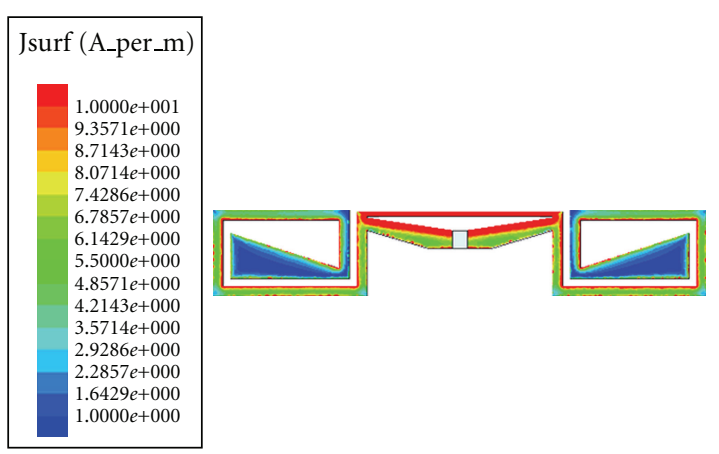

FIGURE 4: Simulated magnitude of complex surface density on the test antenna at $900 \mathrm{MHz}$.

3.2. Selective Deposition Masks. To identify the areas in need for a thicker ink layer, the surface current density on the tag antenna was plotted, in Figure 4, at the tag's optimal operating frequency.

At this stage, there are many options on how to form the selective deposition masks. The main problem is to define the current density level which justifies increasing the ink layer. Selecting an inadequate level will lead to increased ink consumption, while selecting a level too high will lead to poor performance. In this study, the problem has been addressed so that the surface current density level at the center of the dipole arm ends (dark blue areas in Figure 4) was used as a reference level.

To test which surface current level is adequate for optimizing the cost-performance relation, three different selective deposition masks were created. First of these masks, called 5 mask, was created by plotting the complex surface currents that exceed the reference surface current density level by $500 \%$. The final two masks were created similarly using 1000\%, 10 mask, and 4000\%, 40 mask, as the relative surface current levels. All three resulting printing masks formed using the simulated surface current levels are shown in Figure 5.

Two additional selective deposition masks were created without EM simulations to verify the effectiveness of the masks created via EM simulations. One of these masks 

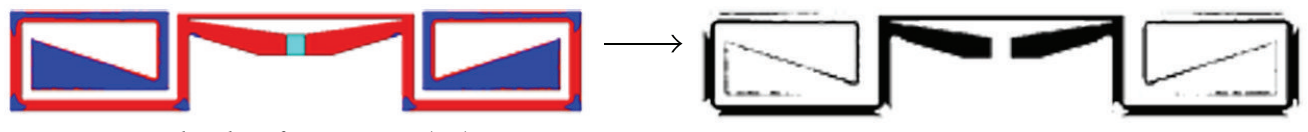

Simulated surface currents (x 5$)$

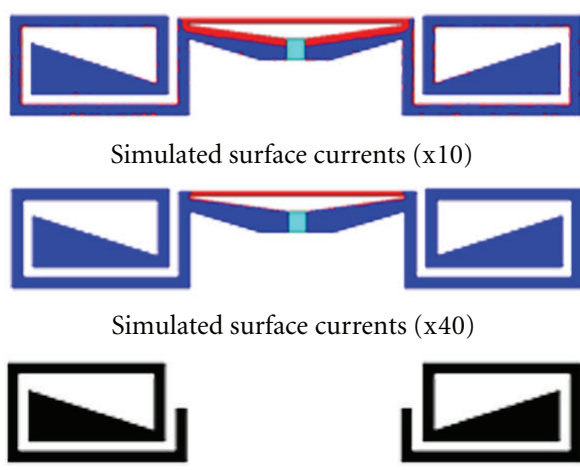

Printing layout (dipole arm increase)

Printing layout (5 mask)

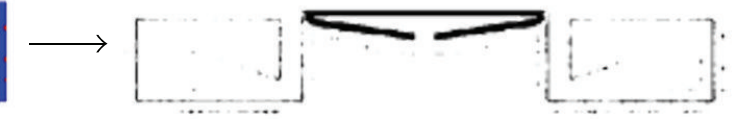

Printing layout (10 mask)
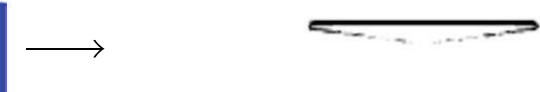

Printing layout (40 mask)

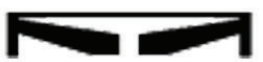

Printing layout (matching network increase)

FIGURE 5: Deposition masks used in this study.

TABle 1: Absolute and relative amount of printed pixels in each deposition mask (1 layer).

\begin{tabular}{lcc}
\hline Deposition mask & $\begin{array}{c}\text { Printed pixels } \\
(\text { abs. })\end{array}$ & $\begin{array}{c}\text { Relative amount of } \\
\text { printed pixels }\end{array}$ \\
\hline Full antenna & $3.95 \cdot 10^{4}$ & $100 \%$ \\
5 mask & $2.31 \cdot 10^{4}$ & $58.4 \%$ \\
10 mask & $6.65 \cdot 10^{3}$ & $16.9 \%$ \\
40 mask & $2.40 \cdot 10^{3}$ & $6.10 \%$ \\
$\begin{array}{l}\text { Dipole arms (DA) } \\
\text { Matching network }\end{array}$ & $3.05 \cdot 10^{4}$ & $77.4 \%$ \\
$($ DM) & $9.27 \cdot 10^{3}$ & $23.6 \%$ \\
\hline
\end{tabular}

increases the ink thickness on the dipole arms only, while the other increases the ink layer only in the matching network area. The matching network increase mask also serves as a good example of a case in which the selective deposition is made based on assumption that high surface currents are found on the impedance-matching network.

Table 1 lists the amount of printed pixels, at $282 \mathrm{dpi}$ which is used as the printing resolution of the samples, and the relative difference in total amount of pixels compared against the full increase case. As it is seen from Table 1, the selective deposition masks bring significant savings in the manufacturing costs by reducing the amount of printed pixels to a small portion of the amount required to print the full antenna pattern.

3.3. Printing Process Parameters. Tag antenna samples were printed by Dimatix DMP-2800 inkjet material printer [25] equipped with $10 \mathrm{pl}$ print head nozzles. Harima NPS-JL silver nanoparticle ink [15] was used as the conductive ink. The printing resolution was set to $282 \mathrm{dpi}, 90 \mu \mathrm{m}$ drop spacing as the mean drop size on the Kapton HN200 was found to be around $120 \mu \mathrm{m}$. The substrate, Kapton HN200, was cleaned with isopropyl alcohol before printing. After printing, the samples were cured for 40 minutes at $200^{\circ} \mathrm{C}$ between layers.

The workflow of the process involving selective deposition was as follows. First the full antenna patterns were printed on the substrate and cured. Samples were brought back from the oven, and additional ink was applied using the selective deposition masks. Finally, the samples were brought back for curing.

The full antenna structure was printed using one to five layers of ink, while the specific deposition masks were printed using one to four layers onto a single layer full antenna structure. Therefore, for example, samples printed using two full antenna layers, and single specific deposition masks should share similar maximum thicknesses.

3.4. Results. The thickness of the inkjet-printed layer was measured optically through cross-sectional images. The thickness per printed layer was found to be around $1.4 \mu \mathrm{m}$. Therefore, the printed antennas have thicknesses ranging from $1.4 \mu \mathrm{m}$ to $7.0 \mu \mathrm{m}$. Figure 6 shows a cross-sectional image from a sample printed with five ink layers.

However, the thickness of the ink layer depends on the printing direction line width of the conductor, and, therefore, thickness varies significantly in a given sample in different areas.

The obtained thickness results agree with other inkjetrelated research publications, for example, in $[26,27]$, that show that the typical thickness per layer is between $0.5 \mu \mathrm{m}$ and $1.5 \mu \mathrm{m}$ if no surface treatment is made for the substrate material. Using $5 \mu \Omega \cdot \mathrm{cm}$ as the mean resistivity for the NPSJL ink, the printed tag antenna sample thicknesses, expressed in terms of skin depths, range from 0.4 to 1.9.

Figure 7 shows microscopic images taken from the surface of the inkjet-printed tag antenna samples. Figures $7(\mathrm{a})$ and 7(b) show the quality of the printed surface with one and four layers of ink. These images show that the surface becomes smoother as more layers are added. Figures $7(c)$ and 


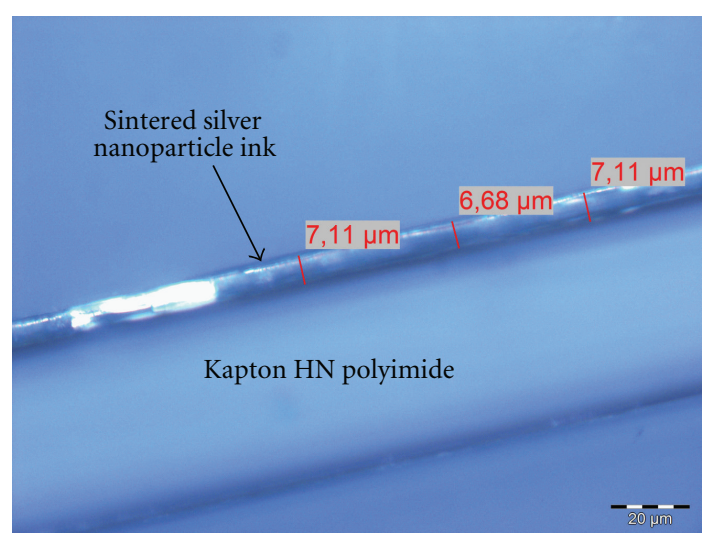

FIGURE 6: Cross-sectional image from an inkjet-printed tag antenna printed using five ink layers. The measured mean thickness of the silver ink layer is $6.97 \mu \mathrm{m}$.

7(d) show examples from surfaces which have additional ink layers selectively deposited using one and four layers.

Using selective deposition to add ink on top of the existing ink surface does not change the dimensions of the tag antenna if the alignment of the additional layer is done properly. It was also verified optically that the ink does not spread excessively when new layers are added using the printing parameters described in Section 3.3.

Theoretical read range was selected as the main tag performance metric in this study. Theoretical read range is the maximal read range, that is, maximum tag to reader antenna distance at which the tag is still able to gather enough power to provide a valid response in free-space conditions. This performance is affected by both the RFID IC sensitivity level as well as the tag antenna radiation properties. As the IC properties remain constant in the measurements performed in this study, any changes in the read range can be considered due to the alternated operation characteristics of the tag antenna.

The theoretical read ranges of the fabricated samples were measured using Tagformance measurement equipment [28] in a compact anechoic UHF RFID measurement chamber. A linear patch-type antenna was used as the reader antenna with a $0.45 \mathrm{~cm}$ separation to the tag under test. Tagformance measurement system calculates the theoretical read range based on the measured path-loss and threshold power, that is, transmit power level at the generator output required to activate the tag under test. This calculation is made according to the following equation:

$$
d_{\mathrm{Tag}}=\frac{\lambda}{4 \pi} \sqrt{\frac{\mathrm{EIRP}}{P_{\mathrm{TS}} L_{\mathrm{fwd}}}},
$$

where EIRP is the maximal allowed equivalent radiated power by the regulations (3.28 W in Europe [29]), $P_{\mathrm{TS}}$ is the threshold power of the tag under test, and $L_{\mathrm{fwd}}$ is the measured path loss in the forward link in the measurement setup.

The measured theoretical read range of each deposition mask and layer quantity is shown in Figure 8. Table 2 lists the
TABLE 2: Ink consumptions to form the deposition masks and their maximal achieved read ranges.

\begin{tabular}{lcc}
\hline Deposition mask & $\begin{array}{c}\text { Ink consumption } \\
\text { to form the mask }\end{array}$ & $\begin{array}{c}\text { Overall achieved } \\
\text { peak read range }\end{array}$ \\
\hline Full antenna & $0.40 \mu \mathrm{L}$ & $7.2 \mathrm{~m}$ \\
5 mask & $0.23 \mu \mathrm{L}$ & $6.8 \mathrm{~m}$ \\
10 mask & $0.06 \mu \mathrm{L}$ & $5.1 \mathrm{~m}$ \\
40 mask & $0.02 \mu \mathrm{L}$ & $4.0 \mathrm{~m}$ \\
Dipole arms & $0.30 \mu \mathrm{L}$ & $3.5 \mathrm{~m}$ \\
Matching network & $0.09 \mu \mathrm{L}$ & $4.6 \mathrm{~m}$ \\
\hline
\end{tabular}

achieved peak read ranges of each deposition masks as well as the total ink consumption required to print the masks using $10 \mathrm{pl}$ print nozzles.

The deposition mask in which the full antenna is printed multiple times shows great performance increase as additional layers are added. The peak read range rises from 3.2 meters ( 1 layer) to 7.2 meters ( 5 layers), equaling a relative increase of $125 \%$.

The selective deposition masks also show great gains in the peak range of the tag although the increase in the amount of printed pixels is significantly lower than in the case in which the full antenna was printed. Samples printed with the selective deposition mask 5 mask nearly match the performance of the full antenna increase mask. In this case the peak read range rises monotonically from 3.9 meters ( 1 layer) to 6.8 meters (4 layers), a $74 \%$ increase.

Samples printed with the 10 mask also show great read range improvements. The maximal read range rises monotonically from 4 meters to 5.1 meters, a $27.5 \%$ increase in read range. In this case, the data shows that the maximal read range has already saturated between samples printed with four and five layers.

Samples made with selective deposition mask 40 mask show the smallest increases in their read ranges between layers. In this case, the read ranges increase monotonically from 3.4 meters to 4 meters, an $18 \%$ relative increase. The measured results show that 40 mask has the potential to provide further gains in the peak read range if more layers are added.

The reference selective deposition masks dipole arm increase and matching network increase show mixed performances. The read range results acquired with samples printed with the dipole arm increase actually decrease slightly as more layers are added. The decrease in the read range is likely to be caused by variations in the RFID IC sensitivities or in the IC-antenna joint. Thus, it can be considered that the read range of the dipole arm increase samples remains constant regardless of the amount of ink layers. The peak read range starts at 3.5 meters (1 layer) and drops down to 3.4 meters (4 layers). These results show clearly that increasing the ink layer thickness only in the dipole arms is highly inefficient.

Samples printed with the matching network increase, however, show monotonically increasing peak read ranges. In this case, the peak read range starts at 3.2 meters and rises 


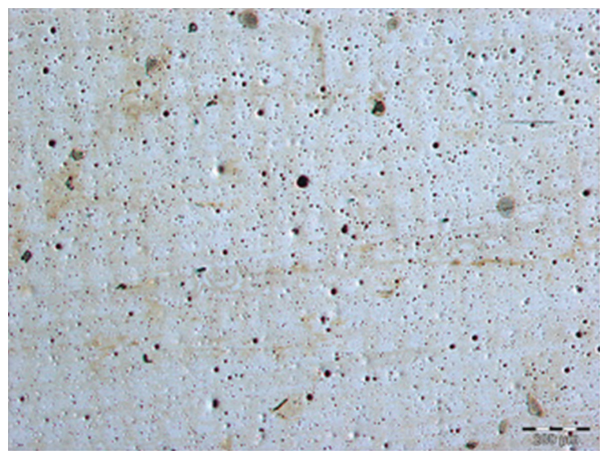

(a)

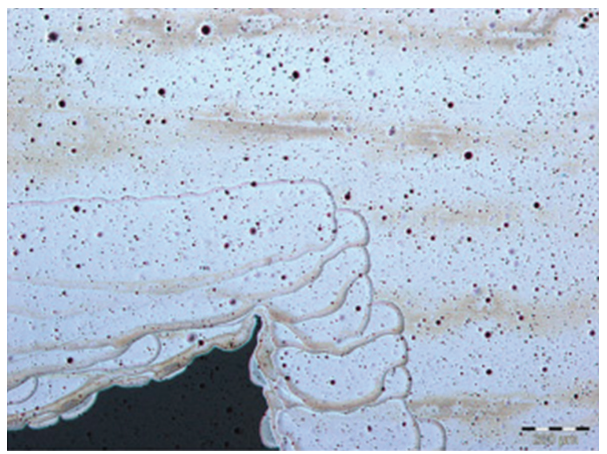

(c)

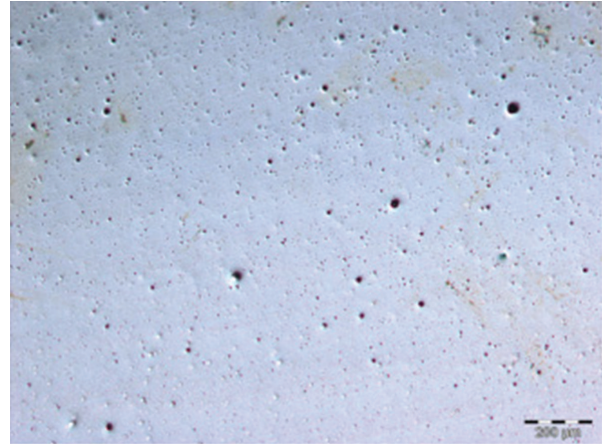

(b)

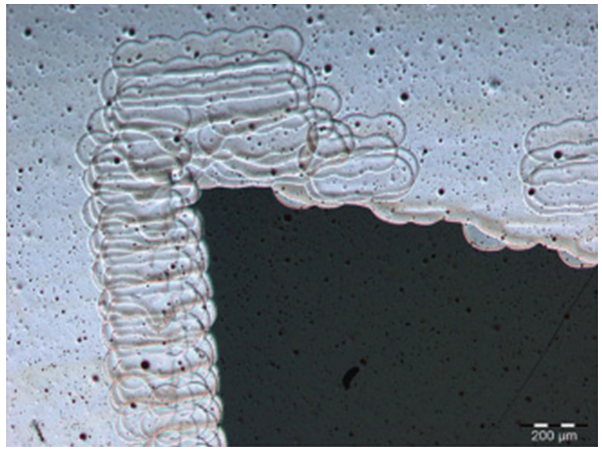

(d)

FIGURE 7: Microscopic images from the inkjet-printed tag antennas. (a) Surface of a sample printed with a single layer. (b) Surface of a sample printed with four full layers. (c) Selective ink deposition with one layer. (d) Selective deposition with four layers.

to 4.6 meters, a relative increase of $43.8 \%$. The performance of these samples is starting to saturate between three and four layer samples. This result shows that moderate read range gains are possible by printing the area around the impedance matching network without any EM simulation optimization.

To examine the cost performance of each deposition mask, the peak read range results of each sample need to be plotted against their ink consumption (uncured volume). The peak read ranges of each sample are plotted as a function of their ink consumptions in Figure 9. In Figure 9, the measured peak ranges of each sample are indicated by round markers. The solid lines represent second-order polynomial fits that describe the trend of the read range behavior as layers are added. Dashed lines represent the estimated performance increase if more layers were to be added to selected deposition masks.

Judging from the data on Figure 9, each of the selective deposition masks acquired through EM simulations shows greater performance-to-cost ratio than the full increase mask. Considering only their efficiencies, 10 mask is clearly the most efficient deposition mask as it produces relatively long read ranges with low ink consumption. The most inefficient method is to add ink to the dipole arms only.

One of the interesting points in Figure 9 is the estimated future trend of 5 mask. According to the projected performance of 5 mask, a tag antenna printed using such selective deposition could match the peak read range of a tag antenna printed using full antenna pattern while providing cost savings.

Figure 10 shows the calculated performance to cost ratio of each deposition mask. The ratio in this case is calculated by dividing the peak read range by the total volume of ink needed to form the resulting antenna.

The performance-to-cost ratios reveal the deposition masks which are the most efficient in terms of producing long read ranges with a set volume of ink. The selective deposition masks made through EM simulations are all showing greater performance-to-cost ratios, after two printed layers, than the full increase mask. Also, the matching network increase shows good ratios, exceeding even the 5 mask in each layer quantity. The poor performance of the dipole arm increase is again highlighted by this graph. 10 mask is showing greater performance to cost ratio than the full increase mask at every amount of layers.

Table 3 lists the possible savings in ink consumption per tag antenna at different performance criteria. The data presented is based on Figure 9. For example, if a read range of five meters is only needed, it can be achieved by printing a single full antenna layer followed by three layers made with 10 mask. Such printing process would nearly half the ink consumption compared to the case where the full antenna would have to be printed using three full layers. In Table 3, the seven meter performance criteria result is based on the projected performance of a 5 mask sample with five layers of ink. 

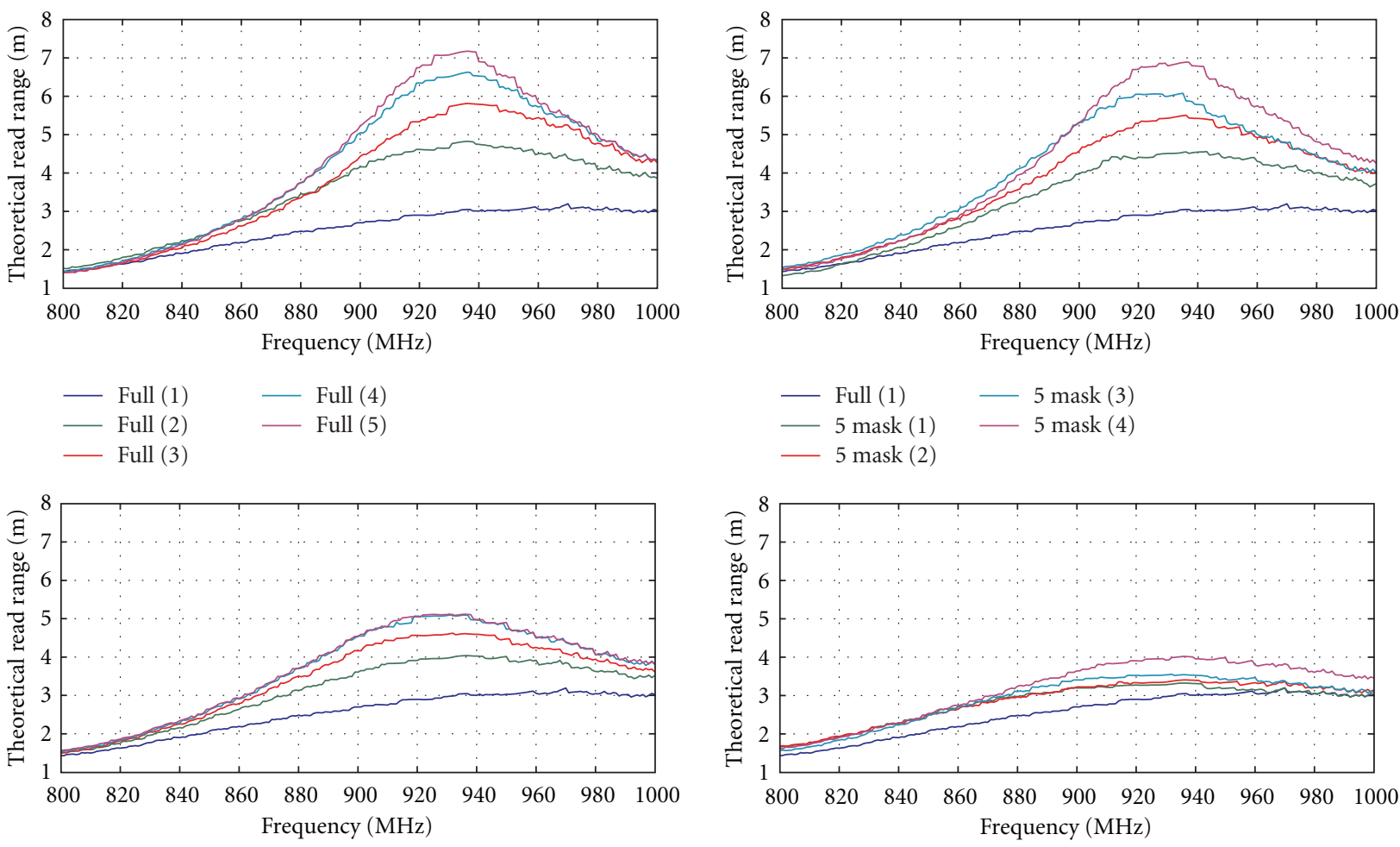

- Full (1) $\quad-10$ mask (3)
-10 mask (1) $\quad 10$ mask (4)
-10 mask (2)

$\begin{array}{ll}- \text { Full (1) } & -40 \text { mask (3) } \\ -40 \text { mask (1) } & -40 \text { mask (4) } \\ -40 \text { mask (2) }\end{array}$
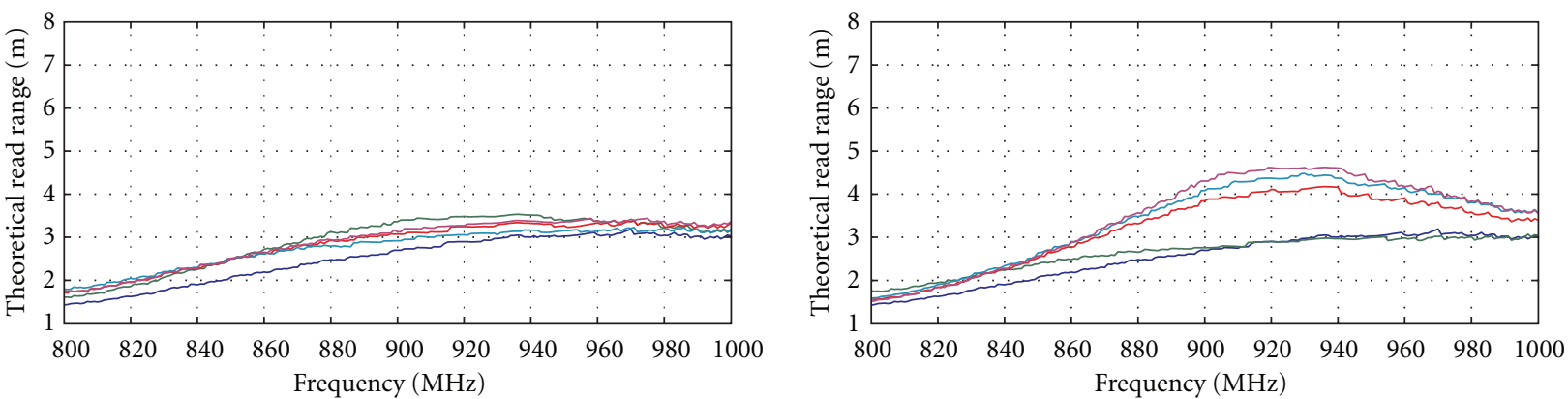

$\begin{array}{ll}\text { Full (1) } & -\mathrm{DA}(3) \\ -\mathrm{DA}(1) & \mathrm{DA}(4)\end{array}$

$\begin{array}{ll}\text { Full }(1) & -\operatorname{DM}(3) \\ -\mathrm{DM}(1) & \mathrm{DM}(5)\end{array}$

FIgURE 8: Measured theoretical read ranges of each deposition mask.

TABLE 3: Ink savings at different tag performance criteria.

\begin{tabular}{lcc}
\hline $\begin{array}{l}\text { Performance } \\
\text { criteria }\end{array}$ & $\begin{array}{c}\text { Selected deposition } \\
\text { mask }\end{array}$ & $\begin{array}{c}\text { Possible savings in } \\
\text { ink consumption }\end{array}$ \\
\hline $7 \mathrm{~m}$ & 5 mask (projected) & $22.1 \%$ \\
$6 \mathrm{~m}$ & 5 mask & $31.1 \%$ \\
$5 \mathrm{~m}$ & 10 mask & $49.8 \%$ \\
$4 \mathrm{~m}$ & 10 mask & $41.6 \%$ \\
\hline
\end{tabular}

The results in Table 3 show that the use-selective deposition masks can greatly drop the ink consumption needed to produce high performance UHF RFID tag antennas. Furthermore, the use of selective ink deposition speeds up the fabrication process as the printer is able to skip the empty pixels in the printing pattern. Therefore, the production costs of inkjet-printed tag antennas can be reduced significantly without any major changes in the production processes or design stages of the antennas.

\section{Discussion of Experimental Results}

The obtained results show that selective deposition can be used to significantly reduce the amount of conductive ink and time to fabricate a passive, high performance, UHF 


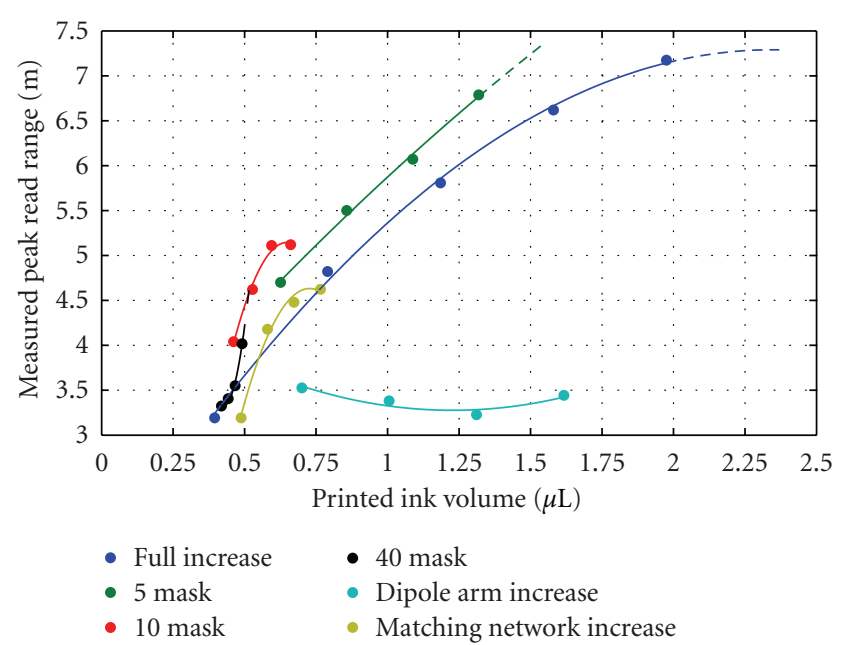

Figure 9: Measured peak read ranges as a function of ink consumption (uncured volume).

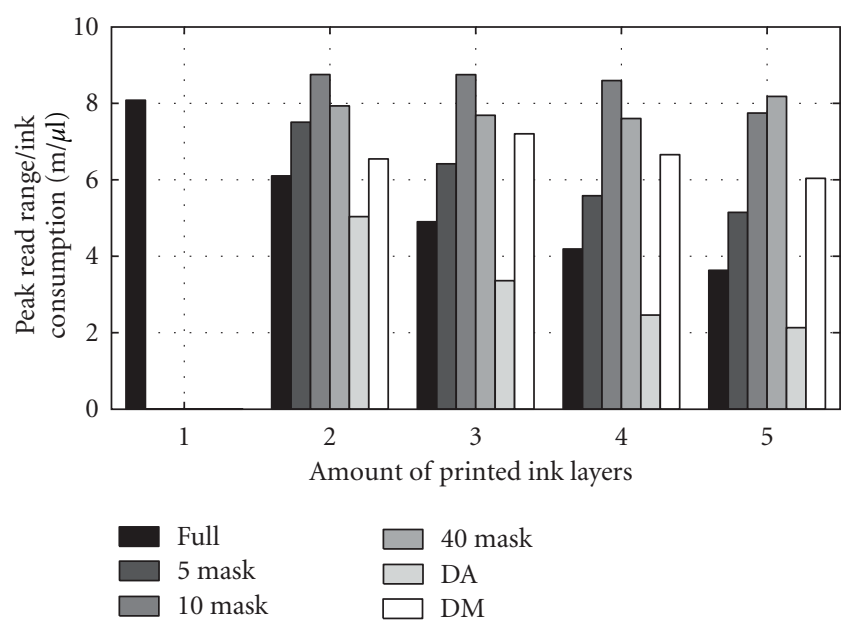

FIgURE 10: Peak read range to ink consumption ratios.

RFID tag antenna using inkjet printing. The impedance matching between the tag antenna and IC remains unaffected by the use of selective deposition. This shows that selective deposition can be used with arbitrary planar tag antenna designs without considering nonuniform ink layer thickness in their design stage.

Read range wise, the samples printed with the full antenna pattern using multiple layers produced the highest read ranges with the amount of ink layers used in this study. None of the samples printed with selective deposition neither matched nor reached the read range of the samples printed using the full antenna pattern. However, if more ink layers were to be added, the performance of the samples printed with 5 mask could match and even exceed the read range levels of the full increase case. This suggests that tag arbitrary antennas printed with selective deposition can achieve similar maximal read ranges as tags which are printed using full layers if the optimal surface current level is used as the printing mask.
Of the three selective deposition masks created via simulations, the one, which adds ink to areas exhibiting ten times higher surface current density than the dipole arm end areas, 10 mask, has the greatest performance to cost ratio. Therefore, in the case of the test antenna, the best costperformance ratio is achieved when ink is added to the inner portions of the impedance-matching network and dipole arms.

The selective deposition mask not created via simulations and that adds ink only to the impedance-matching network area in the tag antenna performs reasonably well in terms of cost performance. Thus, such selective deposition is advised if no simulation tools are available for further costperformance optimization.

\section{Conclusions}

This paper has presented a selective ink deposition method for performance-cost optimization of inkjet-printed UHF RFID tag antennas. The performance-cost optimization in this study has been made by identifying the areas on the tag antenna which exhibit high surface current density levels. A high-resolution printing mask is then created based on these surface currents. The printing mask selectively adds conductive ink only to these areas, possibly allowing for significant savings in the ink consumption and time required to print a high-performance tag antenna.

The results shown in this paper demonstrate the great potential of selective deposition masks acquired through simulations to reduce ink consumption while maintaining adequate performance levels. Moreover, these results show that it should be possible to match the read range distances of a case in which the tag antenna is printed using the full antenna pattern by using the selective deposition technique.

In terms of savings in ink consumption, the selective deposition masks showed decreases between 22\% and 50\% in the required ink volume against the full antenna pattern increase in case of the dipole-type tag antenna. These savings are possible between attainable read ranges of three to seven meters. Even greater ink savings and attainable read ranges could be achieved by discovering the optimal surface current level used as the selective deposition mask.

Future work involving selective deposition is how to provide further improvements in the performance-to-cost ratio. One of the possible approaches is to use different selective deposition masks in the fabrication of a single tag antenna.

\section{Acknowledgments}

This research work was funded by the Finnish Funding Agency for Technology and Innovation (TEKES), the Academy of Finland, the Centennial Foundation of Finnish Technology Industries, the Finnish Foundation for Technology Promotion (TES), and KAUTE foundation. The authors would like to thank Jukka Lindfors and Marko Silvennoinen from Tampere University of Technology, Optoelectronics 
Research Center for their help with the thickness measurements of the ink layer.

\section{References}

[1] H. C. Jung, S. H. Cho, J. W. Joung, and Y. S. Oh, "Studies on inkjet-printed conducting lines for electronic devices," Journal of Electronic Materials, vol. 36, no. 9, pp. 1211-1218, 2007.

[2] S. Molesa, D. R. Redinger, D. C. Huang, and V. Subramanian, "High quality inkjet-printed multilevel interconnects and inductive componentson plastic for ultra-low-cost RFID applications," Materials Research Society Symposium Proceedings, vol. 769, pp. 831-836, 2003.

[3] Z. Konstas, A. Rida, R. Vyas, K. Katsibas, K. N. Uzunoglu, and M. M. Tentzeris, "A novel "Green" inkjet-printed Z-shaped monopole antenna for RFID applications," in Proceedings of the 3rd European Conference on Antennas and Propagation (EuCAP '09), pp. 2340-2343, March 2009.

[4] J. Mujal, E. Ramon, E. Diaz et al., "Inkjet printed antennas for NFC systems," in Proceedings of the 17th IEEE International Conference on Electronics, Circuits, and Systems (ICECS '10), pp. 1220-1223, December 2010.

[5] Y. Amin, S. Prokkola, B. Shao, J. Hallstedt, H. Tenhunen, and Z. Li-Rong, "Inkjet printed paper based quadrate bowtie antennas for UHF RFID tags," in Proceedings of the 11th International Conference on Advanced Communication Technology (ICACT '09), vol. 1, pp. 109-112, February 2009.

[6] Z. Linlin, S. Rodriguez, L. Zhang, B. Shao, and L. R. Zheng, "Design and implementation of a fully reconfigurable chipless RFID tag using inkjet printing technology," in Proceedings of the IEEE International Symposium on Circuits and Systems (ISCAS '08), pp. 1524-1527, May 2008.

[7] P. V. Nikitin, S. Lam, and K. V. S. Rao, "Low cost silver ink RFID tag antennas," in Proceedings of the IEEE Antennas and Propagation Society International Symposium and USNC/URSI Meeting, vol. 2, pp. 353-356, July 2005.

[8] J. Virtanen, L. Ukkonen, T. Björninen, A. Z. Elsherbeni, and L. Sydänheimo, "Inkjet-printed humidity sensor for passive UHF RFID systems," IEEE Transactions on Instrumentation and Measurement, vol. 60, no. 8, pp. 2768-2777, 2011.

[9] V. Lakafosis, A. Rida, R. Vyas, Y. Li, S. Nikolaou, and M. M. Tentzeris, "Progress towards the first wireless sensor networks consisting of inkjet-printed, paper-based RFIDenabled sensor tags," Proceedings of the IEEE, vol. 98, no. 9, pp. 1601-1609, 2010.

[10] M. M. Tentzeris, "Novel paper-based inkjet-printed antennas and wireless sensor modules," in Proceedings of the IEEE International Conference on Microwaves, Communications, Antennas and Electronic Systems (COMCAS '08), pp. 1-8, May 2008.

[11] J. Virtanen, T. Björninen, L. Ukkonen et al., "The effect of conductor thickness in passive inkjet printed RFID tags," in Proceedings of the IEEE Antennas and Propagation Society International Symposium (APSURSI '10), pp. 1-4, July 2010.

[12] J. Siden, T. Olsson, A. Koptioug, and H. E. Nilsson, "Reduced amount of conductive ink with gridded printed antennas," in Proceedings of the 5th International Conference on Polymers and Adhesives in Microelectronics and Photonics, Polytronic, pp. 8689, October 2005.

[13] J. Siden, M. K. Fein, A. Koptyug, and H. E. Nilsson, "Printed antennas with variable conductive ink layer thickness," IET Microwaves, Antennas \& Propagation, vol. 1, no. 2, pp. 401407, 2007.
[14] D. M. Pozar, Microwave Engineering, John Wiley \& Sons, New York, NY, USA, 3rd edition, 2002.

[15] Harima, "NPS-JL Silver Nanoparticle Ink, NanoPaste series, Metal Paste for Thin Film Formation," Datasheet, http://www .harima.co.jp/en/products/pdf/16-17e.pdf.

[16] V. Pynttari, R. Makinen, J. Lilja, V. Pekkanen, P. Mansikkamaki, and M. Kivikoski, "Significance of conductivity and thickness of thin inkjet printed microstrip lines," in Proceedings of the 12th IEEE Workshop on Signal Propagation on Interconnects (SPI '08), pp. 1-4, May 2008.

[17] M. Mantysalo, P. Mansikkamaki, J. Miettinen et al., "Evaluation of inkjet technology for electronic packaging and system integration," in Proceedings of the 57th Electronic Components and Technology Conference (ECTC '07), pp. 89-94, May 2007.

[18] "Alien Higgs-3 RFID IC, Product Overview," http://www.alientechnology.com/docs/products/Alien-Technology-Higgs3-ALC-360.pdf.

[19] DuPont and H. N. Kapton, Datasheet, http://www2.dupont .com/Kapton/en_US/assets/downloads/pdf/HN_datasheet.pdf.

[20] G. Marrocco, "The art of UHF RFID antenna design: impedance-matching and size-reduction techniques," IEEE Antennas and Propagation Magazine, vol. 50, no. 1, pp. 66-79, 2008.

[21] K. Kurokawa, "Power waves and the scattering matrix," IEEE Transactions on Microwave Theory and Techniques, vol. 13, no. 3, pp. 194-202, 1965.

[22] K. V. S. Rao, P. V. Nikitin, and S. F. Lam, "Impedance matching concepts in RFID transponder design," in Proceedings of the 4th IEEE Workshop on Automatic Identification Advanced Technologies, pp. 39-42, October 2005.

[23] T. Björninen, M. Lauri, L. Ukkonen, R. Ritala, A. Z. Elsherbeni, and L. Sydänheimo, "Wireless measurement of RFID IC impedance," IEEE Transactions on Instrumentation and Measurement, vol. 60, no. 9, pp. 3194-3206, 2011.

[24] "Ansys High Frequency Structure Simulator (HFSS) 13," http://www.ansys.com/.

[25] "Fujifilm, Dimatix DMP-2800 Materials Printer, Overview," http://www.fujifilmusa.com/products/industrial_inkjet_printheads/deposition-products/dmp-2800/index.html.

[26] A. K. Sowpati, V. K. Palukuru, V. Pynttäri, R. Makinen, M. V. Kartikeyan, and H. Jantunen, "Performance of printable antennas with different conductor thickness," Progress in Electromagnetics Research Letters, vol. 13, pp. 59-65, 2010.

[27] M. Mantysalo and P. Mansikkamaki, "An inkjet-deposited antenna for $2.4 \mathrm{GHz}$ applications," AEU International Journal of Electronics and Communications, vol. 63, pp. 31-35, 2007.

[28] Voyantic Ltd., Tagformance Lite, Measurement Unit Overview, http://www.voyantic.com/index.php?trg=browse \& id=130.

[29] GS1, "Regulatory Status for Using RFID in the UHF Spectrum," http://www.gs1.org/docs/epcglobal/UHF_Regulations.pdf. 

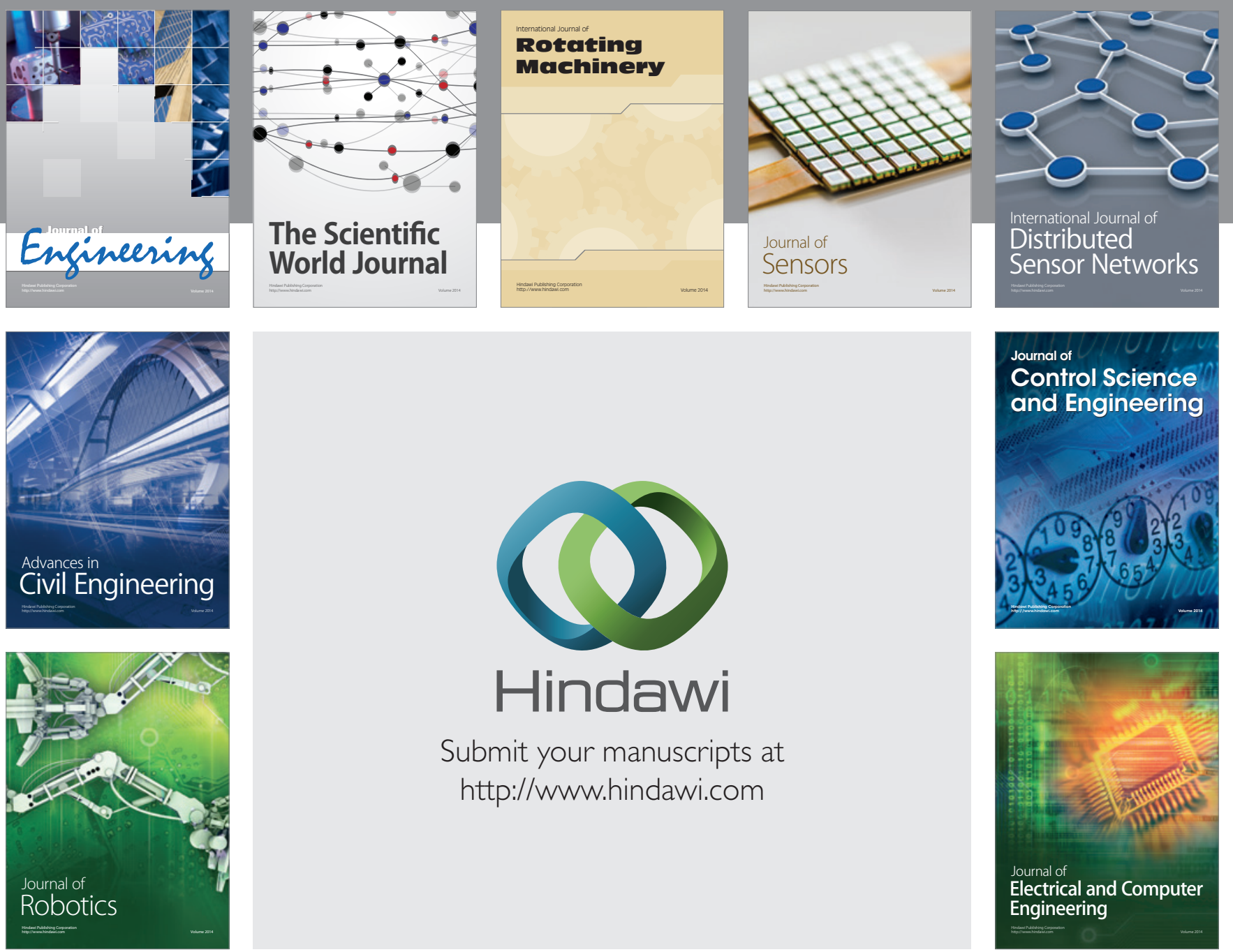

Submit your manuscripts at

http://www.hindawi.com
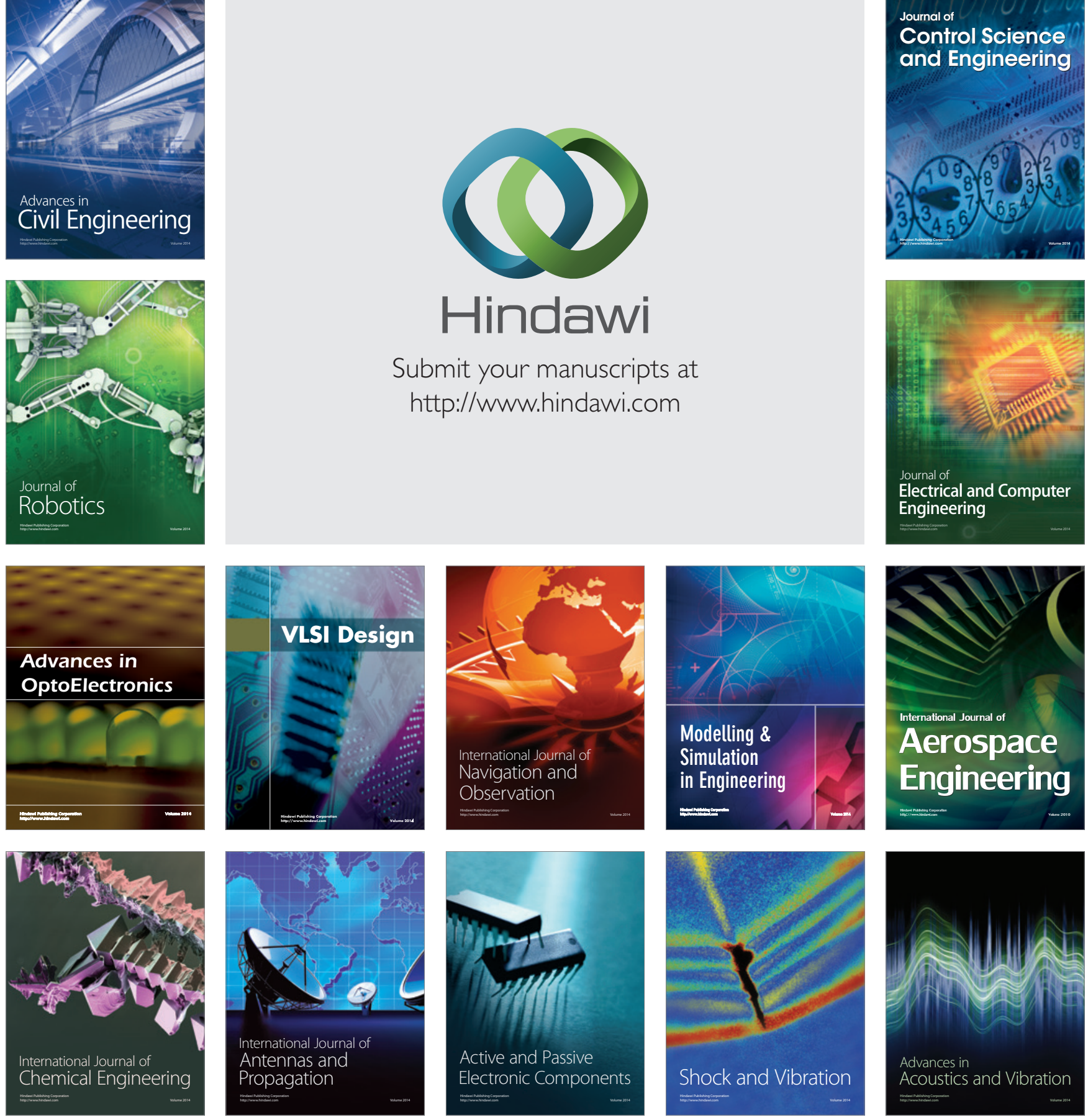